converge to a function $\phi(z)$ analytic in $S$ and such that $|\phi(z)|<1$ for $z$ in $S$. Since the original sequence $\left\{p_{n}(z)\right\}$ converges by hypothesis in $R$, the limit function of the subsequence would necessarily agree with $f(z)$ in $R$. But $\phi(z)$ could not be identical with $f(z)$ in $R$, for then $\phi(z)$ would have to equal unity at interior points of $S$, namely, at the boundary points of $R$ which lie interior to $S$.

It would appear from this remark that Theorem 1 (and Theorem 2 as well) might admit of extension to an arbitrary finite simply connected region whose complete boundary is also the boundary of an infinite region, and that a modification of the methods used in the proofs of the present paper would serve to establish such an extension. The writer hopes to answer this question in a later paper.

Union College,

Schenectady, N. Y.

\title{
NOTE ON A SIMPLE TYPE OF ALGEBRA IN WHICH THE CANCELLATION LAW OF ADDITION DOES NOT HOLD
}

\section{BY H. S. VANDIVER}

1. Introduction. I do not imagine that the algebraic system considered in this note can be new, but if it has been overlooked this has probably happened because of its simplicity. However, we shall be most interested here in examining the connection of the system with the foundations of ordinary algebra. As we shall see, the symbols employed have most of the properties of rational integers, the principal exceptions being that they are finite in number and from

$$
a+b=a+c
$$

we cannot infer $b=c$ in general.*

2. Description of the System. Suppose we introduce the natural numbers $1,2,3, \cdots$, employing for their use Peano's system

* In a system in which we may always infer $b=c$ under the condition given we shall say the cancellation law holds. 
of axioms. Instead of building up ordinary arithmetic by considering the operations of addition and multiplication on the natural numbers, we may introduce symbols

$$
C_{1}, C_{2}, C_{3}, \cdots \text {, }
$$

a relation symbo.ized by $(+)$ and another by $(=)$, and such that

$$
C_{1}+C_{1}=C_{2}, \quad C_{2}+C_{1}=C_{3}, \cdots, C_{n}+C_{1}=C_{n^{\prime}}, \cdots,
$$

where $n$ denotes a natural number and $n^{\prime}$ its immediate successor in the set of natural numbers. We also write

$$
C_{m}+C_{n^{\prime}}=\left(C_{m}+C_{n}\right)+C_{1},
$$

and by employing equality axioms and induction we obtain from this the associative and commutative laws of addition, involving the $C$ 's in a similar manner to that, for example, employed by Pringsheim.*

We introduce an operation, multiplication, such that

$$
C_{m} C_{n^{\prime}}=C_{m} C_{n}+C_{m}, \quad C_{m} C_{1}=C_{m},
$$

and employing these we may prove by induction, as did Pringsheim, the distributive law, the associative law, and the commutative law of multiplication involving the $C$ 's.

All the above mentioned arguments are independent of any possible equalities among the $C$ 's. If we assume that $C_{m}=C_{n}$ if and only if $m$ and $n$ denote the same natural numbers, then consider the relations

$$
C_{i}+C_{j}=C_{k} \quad \text { and } \quad C_{i} C_{j}=C_{m} .
$$

Suppose we replace any such relations by

$$
i+j=k \quad \text { and } \quad i j=m \text {; }
$$

then we have an example of isomorphism, there being a one-toone correspondence between the subscripts of the $C$ 's and the natural numbers themselves under addition and multiplication.

This gives the elementary arithmetic of the natural numbers. We shall employ, for convenience, this arithmetic in what follows, although the system considered is really independent of it.

*Vorlesungen über Zahlen und Funktionenlehre, vol. 1, part 1, pp. 4-30. 
Let us assume now that $C_{1}, C_{2}, \cdots, C_{j}$ are distinct, but $C_{i}=C_{j+1}$ with $j+1>i$; then in the set (1) there are just $j$ distinct elements

$$
C_{1}, C_{2}, C_{3}, \cdots, C_{i}, \cdots, C_{j}
$$

since

$$
C_{j+1}=C_{i} \quad \text { and } \quad C_{j+2}=C_{i+1},
$$

and $i+1 \leqq j+1$. To indicate a certain property of the set (2) we shall consider a special case. Suppose that $C_{1}, C_{2}, \cdots, C_{6}$ are distinct and that $C_{7}=C_{4}$; then from $C_{6}+C_{1}=C_{3}+C_{1}$ we cannot infer $C_{6}=C_{3}$; that is, the cancellation law of addition does not hold in this algebra.

We also see from the way in which it was introduced that this system (2) is just as elementary and natural as ordinary arithmetic. It is obviously not a ring in general. If we take the particular case, however, where $i=1$ in (2), then

$$
C_{j}+C_{m}=C_{m}
$$

for $m=1,2, \cdots, j$, and also

$$
C_{j} C_{k}=C_{j}
$$

so that $C_{j}$ has the properties of a zero element, and the set (2) is isomorphic in this case to a complete system of residue classes, modulo $j$, and forms a ring.

3. Semi-Groups. To derive properties of this finite arithmetic (as well as rings) and to classify it among the different types of abstract algebras it is very convenient to employ the concept of semi-group, which will be defined as a set of elements which is closed under a certain operation symbolized by $O$, such that the associative law holds between the elements of the set combined under this operation; in other words, the set forms a group without the restriction that for $A$ and $B$ in the set there exists an $X$ such that $A \circ X=B$, and a $Y$ such that $Y \circ A=B$. Also the usual equality axioms hold.

If in addition we may infer from

$$
A \circ B=A \circ C \text { and } B \circ D=C \circ D,
$$

the relation 


$$
B=C,
$$

$A, B, C$ and $D$ denoting any elements of the set, then we shall call the set a quasi-group.* We shall now write, in any semigroup, $A B$ for $A \circ B$, and $A^{n}$ for $A \circ A \circ \cdots$ to $n$ terms.

If an element $I$ exists in the semi-group $S$ such that $A I=A$ and $I A=A$ for any $A$ in $S$, then $I$ is called the identity or unity element of $S$. It is easily seen that there cannot be more than one identity element of a semi-group. A unit element of $S$ (containing an identity element) is an element $U$ in $S$ such that there exists a $U^{\prime}$ and $U^{\prime \prime}$ in $S$ for which $U U^{\prime}=I$ and $U^{\prime \prime} U=I$.

An element $C$ of any semi-group $S$ is called cancellable if from $C A=C B$ and $M C=N C$, we may infer $A=B$ and $M=N$ for any $A, B, M$ and $N$ in $S$; otherwise we shall call it non-cancellable.

We shall now prove the following theorem.

THEOREM 1. In order that a finite semi-group contain a cancellable element it is necessary and sufficient that it contain an identity element.

Let

$$
A_{1}, A_{2}, \cdots, A_{k}
$$

be the distinct elements of a finite semi-group $S$ and assume that $C$ is a cancellable element of $S$; then

$$
C A_{1}, C A_{2}, \cdots, C A_{k}
$$

is a permutation of (3), because $C A_{i}=C A_{j}$ gives $A_{i}=A_{j}$, since $C$ is cancellable. Hence for a given $A_{n}$ in (3) there is an $A_{x}$ in (3) such that

$$
C A_{x}=A_{h} .
$$

If $C$ is cancellable, so is $C^{r}$. In the set

$$
C, C^{2}, C^{3}, \cdots
$$

we must have repetitions since the set is finite. Let

$$
C^{t}=C^{t+e},
$$

with $e>0$. If $t>1$, then, since $C^{t-1}$ is cancellable, we obtain

* Dickson, Transactions of this Society, vol. 6 (1905), calls this system a semi-group. 


$$
C=C^{e+1}
$$

so that this relation holds for all cases. Now for a given $h$ consider $A_{x}$ from (4); we shall have from (5)

$$
C A_{x}=C^{e}\left(C A_{x}\right) \text {, }
$$

and

$$
A_{h}=C^{e} A_{h} .
$$

Similarly, considering in lieu of (3a) the set

$$
A_{1} C, A_{2} C, \cdots, A_{k} C \text {, }
$$

we obtain

$$
A_{h}=A_{h} C^{e},
$$

so that (6) and (7) show that $C^{e}=I$ is an identity element of $S$. The condition of the theorem is sufficient since from $A I=A I$ we have $A=A$ and similarly for $I A=I A$.

The relation (4) and its analogon $A_{y} C=A_{h}$ give the known result that any finite quasi-group is a group; hence my introduction of the term quasi.

4. Properties of the System. Consider a finite semi-group given by powers of a single element $A$. It may have the distinct elements

$$
A, A^{2}, \cdots, A^{i}, \cdots, A^{j}
$$

with

$$
A^{j+1}=A^{i} \text {. }
$$

This set is isomorphic with the additive semi-group (2). The elements

$$
A^{i}, \cdots, A^{j}
$$

are known to form a group. (This is conveniently proved by first noting that they form a quasi-group.) Applying this statement to the finite algebra given by (2), we obtain the result that $C_{i}, C_{i+1}, \cdots, C_{j}$ form under addition and multiplication a ring.

Although there may be no zero elements in the set (2), yet 
we may have non-cancellable elements in the semi-group formed by multiplication of the elements. For if $j=6, i=4$, we have

$$
C_{2} C_{2}=C_{5} C_{2},
$$

but we cannot infer

$$
C_{2}=C_{5}
$$

Hence, the system differs from a ring in this respect, for in the latter a non-cancellable element under multiplication is necessarily a zero-divisor.

If, in the relation (2), $i>1$, then there is no zero element in the set, or considered as an additive semi-group there is no identity element in the set; hence, by Theorem 1 there is no cancellable element under addition. This is in sharp contrast to the case when $i=1$, in which case we have a group under addition and all the elements are cancellable.

Considering the multiplicative semi-group of (2) for $i=1$, we have the case where the system is isomorphic with the residue classes, modulo $j$, so that the cancellable elements are known. For $i>1$ there is no cancellable element except $C_{1}$, for the cancellable elements form a quasi-group and therefore a group which includes $C_{1}$; hence, if $C_{a}$ is cancellable, there is a $C_{b}$ such that

$$
C_{a} C_{b}=C_{1},
$$

which is impossible, since $a b>1$ and $i>1$.

The various properties we have established for (2) may be stated as follows.

THEOREM 2. Under addition and multiplication with the assumptions given, the set (2) forms an algebra in which for $i>1$, the additive semi-group of the algebra contains no cancellable elements and the multiplicative semi-group contains no cancellable elements other than $C_{1}$. The elements $C_{i}, C_{i+1}, \cdots, C_{j}$ form a ring which for $i=1$ is isomorphic with the system of all residue classes, modulo $j$.

5. Extensions and Modifications of the System. We may obtain other algebras which have the property that cancellation under addition is not possible in general and multiplication is noncommutative by taking the system of all square matrices of order $n$ whose elements are contained in (2). 
Suppose we consider the natural numbers and consider a relation* between them which we shall call equivalence, symbolized by $(\cong)$, such that if $a$ and $b$ are natural numbers and if $a<i$, then from $a \cong b$ it follows that $a=b$, and if $a$ and $b \geqq i$, then $a \equiv b(\bmod m)$. For $m=j-i+1$, we obtain a system which is isomorphic with the $C^{\prime}$ 's in (2) provided equivalence takes the place of equality, the number of non-equivalent integers being $j$. Using this relation between rational integers, we may consider conditional equivalences, such as

$$
x^{2} \cong a,
$$

where $a$ is a positive integer. The question naturally arises as to possible generalizations of the law of quadratic reciprocity, since equivalence is an extension of the idea of congruence.

6. The System as a Type of Algebra. As to the place of our system among known types of associative algebras in which the usual distributive laws hold, we may first define a ring as a system of elements which form an Abelian group under an operation called addition, a semi-group under multiplication, and in which the right and left hand distributive laws hold. But number theorists have worked since the beginning of the history of their subject in systems which are not always rings, for example, in connection with the problem of expressing a positive integer as the sum of positive cubes. The positive integers under addition form an Abelian quasi-group in lieu of an Abelian group. The next natural generalization is to replace quasi-group by semigroup, and if this is done, our system (2) exemplifies an algebra of the type where the elements form a semi-group under addition, but not a quasi-group.

We may, indeed, proceed further and define a semi-ring or associative algebra as a set of elements forming a semi-group under addition, a semi-group under multiplication, and in which the right and left hand distributive laws hold. This leads us naturally to the consideration of associative algebras in which addition is not commutative, that is, the restriction "Abelian" is removed in connection with the original definitions of addition. Certain of these types I hope to take up in another paper.

The University of Texas

\footnotetext{
* As suggested by A. Church.
} 